\title{
Designing for Mode Shift Opportunity with Metropolitan Scale Simulation
}

\author{
Kanaad Deodhar \\ Colin Laurence \\ Jane Macfarlane* \\ kdeodhar@berkeley.edu \\ colin_laurence@berkeley.edu \\ janemacfarlane@berkeley.edu \\ University of California Berkeley \\ Berkeley, CA
}

\begin{abstract}
Shifting vehicle drivers to alternate modes is becoming a key focus of city planning groups. Key to understanding how to posit new transit opportunities requires a granular understanding of origindestination travel demand. By using Mobiliti, a HPC simulation developed at Lawrence Berkeley National Laboratory that populates origins and destinations and simulates their use of the transportation network, that granular understanding can be achieved. This data can be used to understand how current and potential future transit routes serve regional demand and how those services can be improved, an increasingly important aspiration in the face of falling transit ridership, increasing congestion, and environmental concerns. To understand how existing transit routes serve the demand, an origin and destination falling within a quarter mile around bus and light rail routes were considered zero-transfer transit-feasible; the results of this rudimentary analysis were noteworthy, with simulated feasible ridership up to five times higher than actual ridership seen by the Santa Clara Valley Transportation Authority. Using OpenTripPlanner, we found the average transit trip time to be approximately three times the driving time for that set of transit-feasible trips. This points to a need to increase the operational efficiency of transit services that cannot compete with auto traffic. The second portion of the analysis focuses on understanding how the transit network can be changed to better accommodate the demand. By understanding the origin-destination pairs unserved by the current transit network, corridors of high demand can be visualized, identified. Public transit is key to cities, and this methodology uses novel data sources to inform the design and development of that oft-overlooked workhorse of urban mobility.
\end{abstract}

\section{KEYWORDS}

Mode Shift, Metropolitan Scale Simulation, Transit

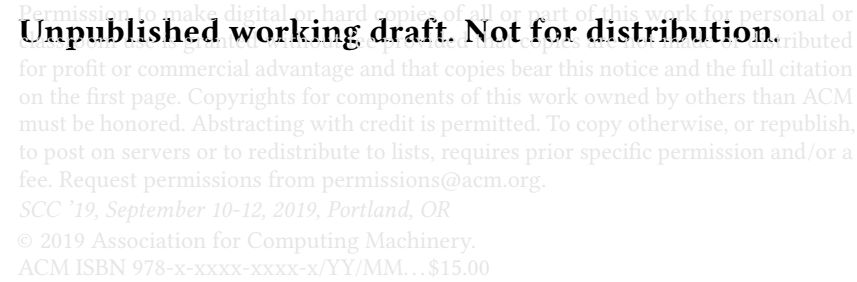

DOI: $10.1145 / 3357492.3358634$

2019-08-01 16:51. Page 1 of 1-6.
ACM Reference Format:

Kanaad Deodhar, Colin Laurence, and Jane Macfarlane. 2019. Designing for Mode Shift Opportunity with Metropolitan Scale Simulation. In Proceedings of SCC 2019: The 2nd ACM/EIGSCC Symposium On Smart Cities and Communities (SCC '19). ACM, New York, NY, USA, 6 pages.

\section{INTRODUCTION}

Throughout the United States, transit ridership has experienced a significant decline in recent years. Due to a combination of insufficient investment, decreasing gasoline prices, a booming economy, and the advent of numerous new mobility services, transit agencies are facing significantly increased competition from other modes. However, it is clear that in order for cities to be the thriving, vibrant economic and cultural centers they were meant to be, there must be a dramatic shift in the transportation culture of the United States.

By using novel data sources that better represent transportation demand, it is possible to understand at a significantly increased granularity how transit networks are being used, and to identify corridors with a high potential for new transit. In combination with other policies, including land use, housing, and urban form, the results of this project will help increase transit's competitiveness in an increasingly complicated transportation network. Santa Clara County is a particularly interesting setting in which to apply these methodologies: the county has very diverse urban topologies, including the large semi-dense metropolitan area of San Jose, a few small towns, vast acreages of parkland, and dozens of technological and academic campuses that create intense, concentrated demand on the transportation network. As one of the counties with the heaviest congestion in the Bay Area, it is critical for the County's economic and social well-being that transportation investment be directed towards increasing modeshift to transit from private vehicles.

To increase public transport usage, the service should be designed in a way that accommodates the levels of service required by customers and by doing so, attract potential users. Some evidence has suggested that some people may not always drive out of necessity, but also by choice [3]. Therefore, it is necessary to promote policies and manage transportation systems in a way that can reduce private transport dependence as well as the need for driving, by providing alternatives to driving. Such policies might involve an improvement in the public transport service (e.g. extension of transit network) to meet consumer needs and expectations. User 
requirements could involve not only proximity of transit stops but also reasonable waiting times [5]. [1] showed that travel time can be considered an advantage of buses and is an important reason for mode choice. Additionally, the time required to park in downtown areas, and the discomfort caused by continuous stop-and-go situations, furthers the advantages of transit.

Work has been done in examining how transit compares with driving within the Bay Area, such as in [4]. This article examines how average commute times vary spatially across the Bay Area in both transit and automobile trips, and how this correlates with various demographic variables. Their origin destination information is similar to ours, as it too uses TAZ pairs provided by the MTC. However, their emphasis was on determining how these times related to job accessibility. We are focused on breaking down individual trips and determining transit feasibility of these trips. In [4], all of the travel times could only be reported at the TAZ level. However, since transit times can vary wildly within a TAZ based model on both proximity to transit stops, and where those transit stops connect to, average travel times at the TAZ level are rather crude for the purpose of examining transit feasibility within a TAZ. Our approach combines GTFS data, TAZ-to-TAZ travel times from Uber Movement, and simulated trips from a high performance computing simulation that allows for large scale modeling of metropolitan areas. This allows us to gain better micro level insights on transit feasibility and effectiveness than have previously been published. The purpose of this paper is to illuminate how the transit network is currently serving transportation demand, examine the competitiveness of transit versus driving, and to identify demands not currently served by transit that are candidates for a next-generation transit offering.

\section{DATA SOURCES}

A variety of data was fused in order to approach this analysis. Core to the analysis is the granular trip leg data. Trip leg data is generated via a parallel discrete event simulation, Mobiliti [2], that is run on high performance computing facilities at Lawrence Berkeley National Laboratory. It uses the Metropolitan Transit Commission's travel demand model, which defines the number of trips between Traffic Analysis Zones (TAZ) and populates origins and destinations inside any given TAZ according to the population density. The result is a highly granular Origin-Destination Matrix across the whole of the nine counties, providing highly-detailed estimations of trips across an entire day. Figure 1 shows the distribution of origins generated from the MTC's CHAMP6 model and a population density model. As a whole, the Mobiliti simulation contains 22 million trips to and from 1.1 million nodes, intersections that act as start and end points. In Santa Clara County, there are around intra-county 6 million trips and 100,000 nodes. Each of these trips contain information on their start and end locations, the travel time, both free flow and congested and the corresponding delay ratio, and the distance traveled.

GTFS: The General Transit Feed Specification is the set of files that define a transit network schedule. The transit agency serving Santa Clara County is the Valley Transportation Authority (VTA), which maintains a network of 81 routes, both bus and light rail.

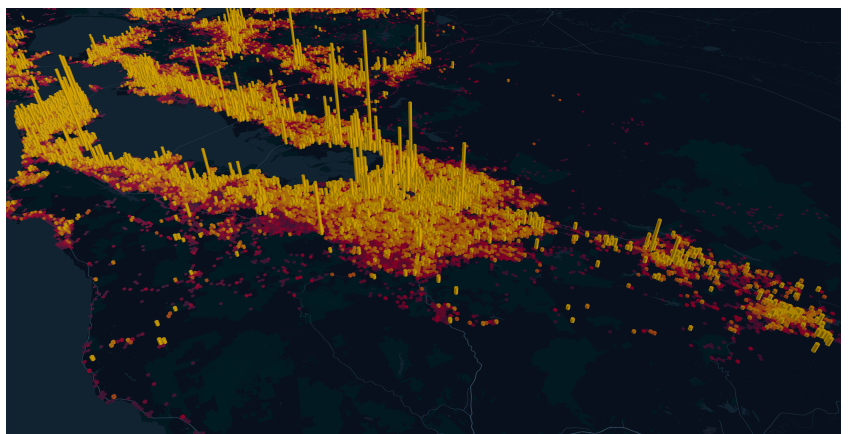

Figure 1: Demand Model Origin Counts - Full Day, Looking NE Over The Bay Area

The data from VTA used in this project included the location of the transit stops, and the routes servicing those stops.

OPENSTREETMAP: The collaboratively edited mapping project that covers the whole world. This network was used in conjunction with the open-source software OpenTripPlanner, which generates modal trip itineraries when given an origin, destination, and time of day.

UBER MOVEMENT: Aggregated average TAZ-to-TAZ travel time data provided by Uber, used to compare the competitiveness of modes.

\section{METHODOLOGIES AND RESULTS}

\subsection{Existing Transit Service}

To understand how the current VTA network is serving demand, each of the 6 million Mobiliti trips inside Santa Clara County was run through OpenTripPlanner, which determined whether or not a given trip could be completed with the existing transit network. The schedule used was that of May 1st, 2019, which was a Wednesday. Along with the schedules, the possibility of using transit for a given trip was determined using a max walk distance of 0.5 miles, which includes walking on either end of the trip and for transfers. For trips for which transit could be used, the number of transfers, total travel time, and waiting time during transfers were recorded. Note that the total travel time includes time spent walking, waiting for transfers, and on transit, but does not include time waiting for the initial transit vehicle. VTA's routes generally have a large enough headway that it is unreasonable to model the passenger arrival rate at the initial transit stop as a Poisson process, so determining the initial wait times would be very difficult. Aggregating these trips by TAZ-to-TAZ pairs and hour of day, and comparing against the Uber Movement data, provides a high-level understanding of general transit competitiveness across Santa Clara County, seen in Table 1.

As expected, as the number of transfers increase, transit competitiveness is dramatically affected: while zero-transfer trips take 4 times as long as a comparable Uber journey, a single transfer causes that ratio to double, to an unacceptable eight-fold increase in travel-time cost. Since single-transfer trips represent the bulk of the feasible trips on the transit network, this is immediate cause for concern, as high utilization of the network is unlikely with such skewed travel time costs. Figure 2 visualizes the daily average 


\begin{tabular}{llll}
\hline $\begin{array}{l}\text { Number of } \\
\text { Transfers }\end{array}$ & $\begin{array}{l}\text { Mean Duration } \\
\text { (mins) }\end{array}$ & Frequency & $\begin{array}{l}\text { Transit-to- } \\
\text { Uber Ratio }\end{array}$ \\
\hline 0 & 21 & 0.26 & 3.89 \\
\hline 1 & 70 & 0.33 & 7.96 \\
\hline $2+$ & 152 & 0.14 & 14.18 \\
\hline Impossible & N/A & 0.26 & N/A \\
\hline
\end{tabular}

Table 1: Trips Broken Down by Number of Transfers Needed to Complete Trip Via Transit

transit-to-uber ratio for each TAZ-to-TAZ pair with more than 25 trips, with red representing a higher ratio. It is immediately apparent that though downtown San Jose and the string of Silicon Valley cities through Palo Alto are generally well-served, many of the outlying regions surrounding San Jose are not. Though it is expected that a sprawling transit network would under perform in a low-density, heterogeneous landscape like Santa Clara County's, examination of high-performing routes provides greater insight.

To understand how individual routes are serving travel demand, a 0.25 -mile walkshed was developed around the ten highest-dailyridership routes in the VTA network, which together account for more than half the daily network ridership. If a Mobiliti trip had both its origin and destination inside this walkshed, and OpenTripPlanner determined that the trip was both feasible by transit and had zero transfers, that trip was considered served as a one-seat journey. By enumerating the trips served, aggregating them by origin TAZ, destination TAZ, and hour-of-day for each route, and then comparing the travel times to the average travel times given by Uber Movement, an understanding of transit competitiveness on specific high-ridership routes can be achieved. Table 2 details these characteristics for those ten routes.

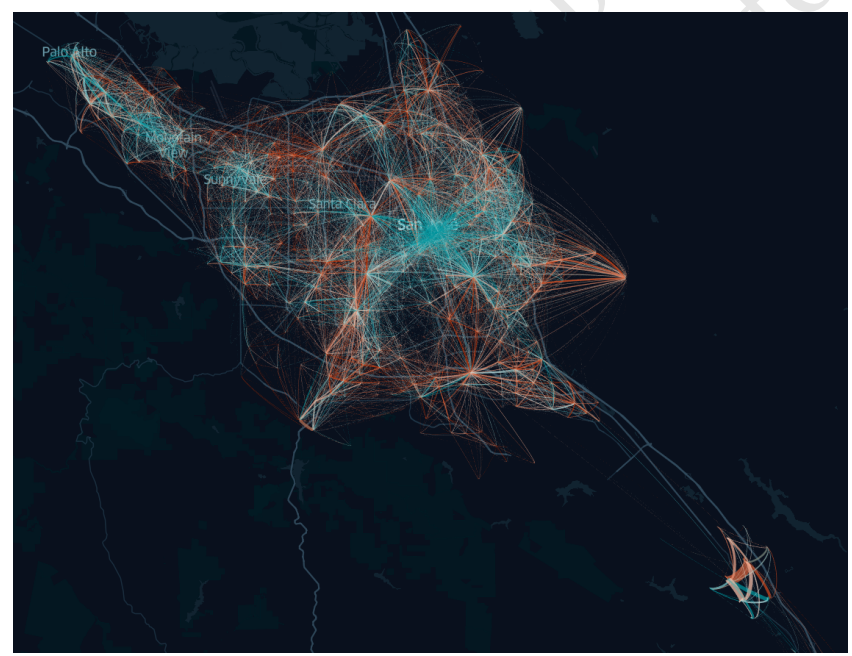

Figure 2: Visualization of Transit-to-Uber Delay Ratios

Immediately, it is clear that there is a massive discrepancy between the real-world and simulated ridership for each of these 2019-08-01 16:51. Page 3 of 1-6.

\begin{tabular}{llll}
\hline VTA Route & $\begin{array}{l}\text { Daily Ridership } \\
\text { (October 2018) }\end{array}$ & $\begin{array}{l}\text { Mobiliti } \\
\text { Ridership }\end{array}$ & $\begin{array}{l}\text { Transit-to-Uber } \\
\text { Travel Time Ratio }\end{array}$ \\
\hline 901 & 16,706 & 27,868 & 3.21 \\
\hline 902 & 12,462 & 19,240 & 3.55 \\
\hline 22 & 9,831 & 71,595 & 2.65 \\
\hline 23 & 6,959 & 36,542 & 3.11 \\
\hline 25 & 6,497 & 26,256 & 3.41 \\
\hline 66 & 5,840 & 33,717 & 3.30 \\
\hline 68 & 4,999 & 24,300 & 3.19 \\
\hline 70 & 4,637 & 17,442 & 3.77 \\
\hline 64 & 3,407 & 23,782 & 3.15 \\
\hline 72 & 3,092 & 20,144 & 2.90 \\
\hline
\end{tabular}

Table 2: Predicted Ridership and Travel Time Comparison for VTA Routes

routes, with one-seat Mobiliti trips up to 5 times the actual daily ridership. Given that the transit travel time was,on average at any given hour of day, 3 times the Uber travel time, it is clear that trip duration is a major factor in driving people away from transit. As these one-seat trips represent the most ideal conditions on the VTA network, a travel time ratio hovering around 3 is far from ideal, though not as catastrophic as the transfer time penalties evident in Table 1. The jump from 21 minutes for a zero transfer trip to 70 minutes for a single transfer trip is only partially due to these trips being a longer distance on average. The median transfer wait time for single transfer trip is 17 minutes and the mean is a full 27 minutes, causing the split between the two very well defined peaks in the transit time histogram in Fig. 3. These lengthy transfer wait times suggest that there is a lack of coordination in transfers in the VTA routes' design.

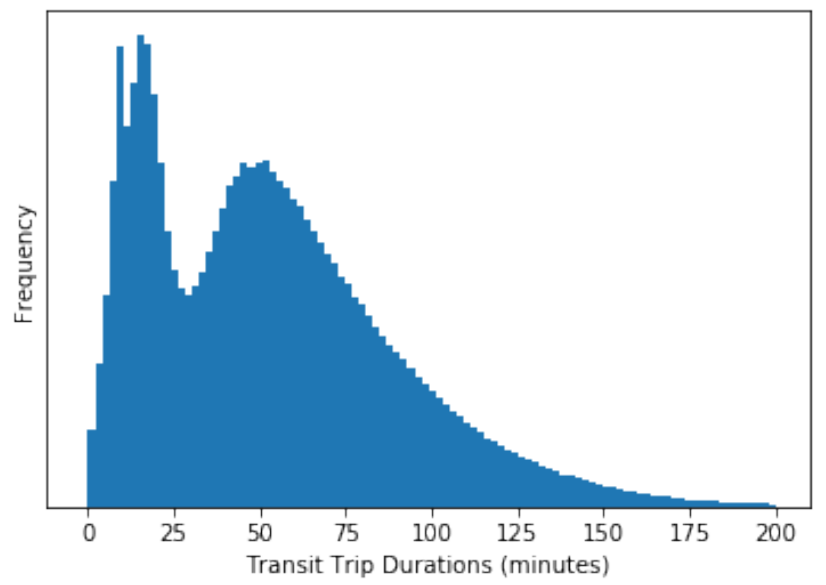

Figure 3: Histogram of Transit Durations for All Trips Possible via Transit 


\subsection{Potential Transit Services}

In addition to examining the existing transit service in terms of overall operating performance and in terms of individual line performance, locations where transit most needs to be expanded were then assessed. To start, a definition of which trips were feasible by transit was created. Ideally, transit trips should be zero transfer. However, given that in Santa Clara County we found that only $26 \%$ of trips could be completed via zero transfer transit, the definition of transit feasible was expanded to also include trips that contain a single transfer with less than 15 minutes of wait time. This definition meant that $39 \%$ were considered transit feasible, with a mean duration of 32 minutes, which was 10 minutes longer than the mean for zero transfer trips. The coordinates for the both the origins and the destinations for these transit feasible trips were then concatenated to give an overall picture of where all users want to go to and from throughout the day. These coordinates of feasible trips are displayed as a heat map in Fig. 4a. Unsurprisingly, in this figure we can see the rough outline of the VTA transit network.

The more significant dataset is the remaining infeasible trips, which consists of single transfer trips with more than 15 minutes of transfer waiting time, all trips with two or more transfers, and trips that cannot be completed using transit. The starts and ends of these infeasible trips are displayed as a heatmap in Fig. 4b. This figure has comparatively little structure, with reasonably high densities of infeasible trips in virtually every corner of the urbanized area. Broadly this suggests that there is room for transit improvement across the entire county, as even areas that have transit services still have plenty of trips that are not served well by transit.

However, this does not help the goal of isolating specific areas for adding transit service. To do this, the trips for which transit is not possible were examined; the heatmap of these trip starts and ends is displayed as Fig. 4c. While this figure is a bit more faint, isolated hotspots can be seen in various locations. The isolation of these hotspots means that these data are well suited for DBSCAN application. DBSCAN is a clustering algorithm that finds continuous areas that meet a given density threshold. Here, this threshold was set to be a minimum of 10,000 daily origins or destinations within a circle of radius 400 meters. Since this dataset was very large, it was first broken down into 20 smaller datasets using KMeans clustering, and DBSCAN was run on each of these 20 KMeans clusters individually. The top 10 largest DBSCAN clusters, are described below in order, along with brief qualitative descriptions. Note that the locations of the clusters are not exact, as DBSCAN clusters can not be properly described by a single point; they inhabit somewhat loosely defined regions. As such, here they are best described by the neighborhoods they cover.

(1) Stanford University, Palo Alto. While there is some bus service to the entrance to Stanford, the campus is so large that most of it is beyond the walkshed. This analysis does not include college operated campus shuttles, only VTA routes, which would improve transit access. However, this result does show that integrating VTA bus services within the campus would bring a large increase in the number of people within walking distance of VTA services.

(2) Industrial along southern end of Zanker Road, Northern San Jose. This industrial park is very large, and currently not served by transit. Presumably it is a large enough job site for it to show up so prominently in the demand profile.

(3) Residential along the western end Santa Teresa Boulevard, Southern San Jose. The eastern end of this road on the far southern periphery of San Jose is served by transit, but it turns north and leaves a quite large residential neighborhood without any service. The cluster is oblong and roughly along this road.

(4) Downtown San Jose. This region is already very well served by transit. It only shows up as a non-served cluster because it is such a major destination that many trips from areas without transit start or end here.

(5) Residential along Clayton Road and Ruby Road, Eastern San Jose. Another large residential neighborhood on the periphery of the city that is not served by transit. The cluster is also oblong and runs roughly along these roads.

(6) Residential in general vicinity of Fairwood Park, Sunnyvale. A quite large residential neighborhood across Calabazas Creek from Mission College, with very limited transit within walking distance.

(7) Residential along western half of Benton Street, Santa Clara. A reasonably high density neighborhood loosely along Benton on either side of the Lawrence Freeway.

(8) Residential near junction of Guadalupe Freeway and Almaden Expressway, San Jose. Slightly unusual cluster, as it includes both residential areas to the west of the freeways, and industrial areas to the east of the freeways.

(9) Oracle Campus and residential area to the north of it, Northern San Jose. This large job site combined with nearby residential areas shows up as a single cluster not served by the VTA. There may be company run shuttles to the Oracle Campus.

(10) Residential along Lick Mill Blvd, Northern San Jose. Almost a continuation of cluster (9), though this cluster is just residential.

This DBSCAN search returned 29 clusters in total which cumulatively contain $11 \%$ of the total origins and destination from trips that can not take transit; the other 19 smaller ones have been omitted for the sake of brevity. The exact number of clusters and, to and extent, their ordering is dependent on the parameters given to DBSCAN. The parameters used in this analysis were determined via trial and error to avoid clusters that were neither the size of a single block nor the size of half a city. However, these 10 neighborhoods are definitely both very poorly served by transit and reasonably dense in terms of origins or destinations of trips. As such, they would be good locations for potential new services.

\section{CONCLUSIONS}

By simulating travel demand at an intersection-granularity, and processing the generated origins and destinations through routefinding software, we can achieve a significant understanding of where existing transit networks are serving, or underserving, the demand. By quantifying the delay faced by transit users in their modal choice, it is evident that even in the best cases, the transit network has three times the travel-time cost of driving. Outside of 


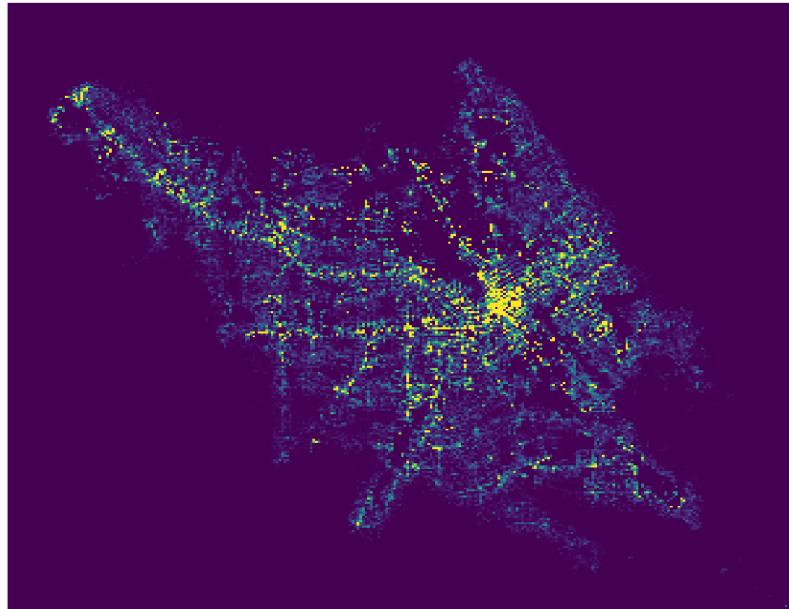

(a) Feasible via transit

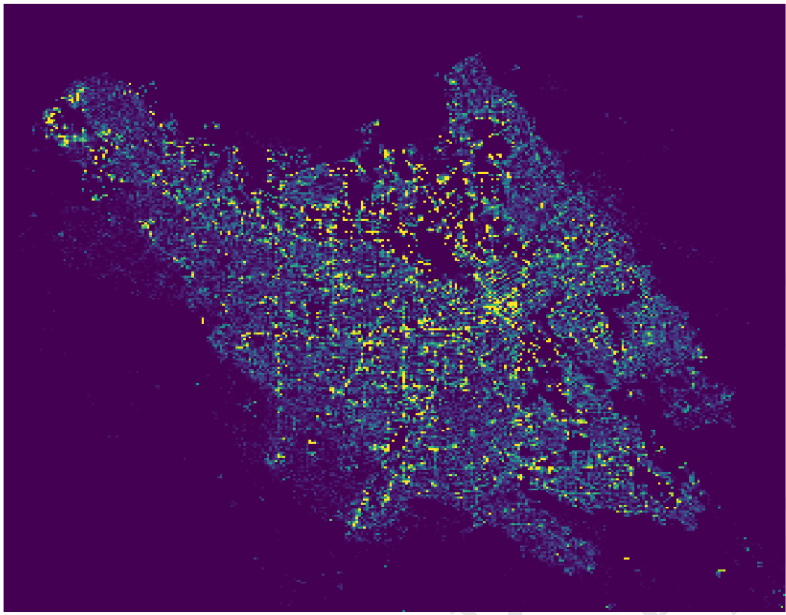

(b) Infeasible via transit

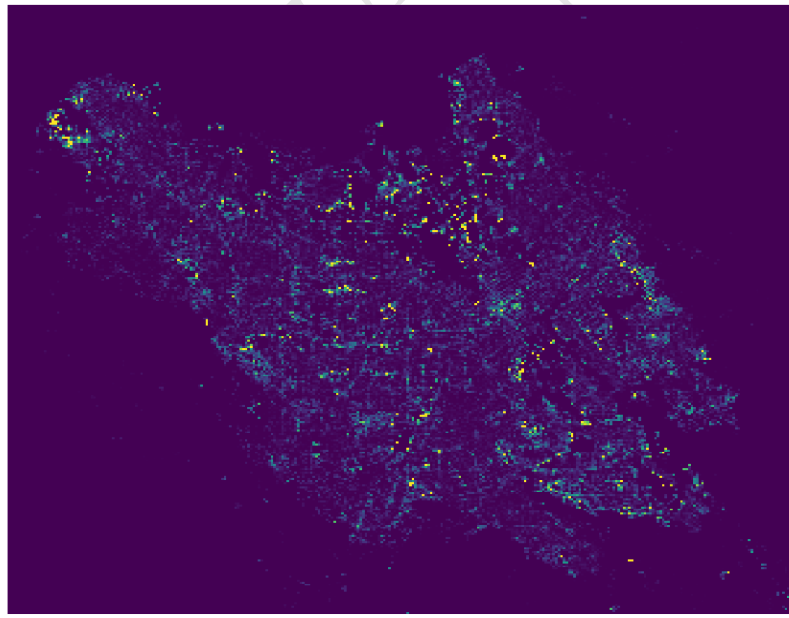

(c) Impossible via transit

2019-08-01 16:51. Page 5 of 1-6.

Figure 4: Concentrations of trip origins and destination throughout the primary urbanized area of Santa Clara county, broken down by transit feasibility. the best cases, which represent the significant majority of transitfeasible trips in the County, the travel-time cost becomes egregious: eight times that of driving for even 1-transfer trips. For transit to become a truly viable mobility option, this number must come down: policies and designs which improve transit competitiveness against single occupancy vehicles, such as dedicated lanes, bus stop rebalancing, coordinated transfers between routes, are crucial for the future of urban mobility options.

Furthermore, we have found that over a quarter of all trips made within Santa Clara County cannot be made using transit assuming a maximum walking distance of 0.5 miles. Promisingly though, we have identified many high density clusters of unserved demand that would be good first steps for expansion of transit coverage. While such service expansions may not necessarily be the most cost effective way to increase ridership, they would be a very cost effective way to increase total coverage, which is a key component of transportation equity.

\section{FUTURE WORK}

One immediate follow up task would be to incorporate proper equity analysis in our recommendations for transit coverage expansion. For example, it would be very useful to know where the largest clusters of unserved demand from low income households are located. Reaching these populations is especially important, as they are less likely to have alternatives to public transit.

In terms of methodology, clustering origins and destinations is immediately helpful in identifying regions of high demand, but treating them separately is also somewhat limiting. To understand how future transit networks can better serve travel demand, it is the trajectories between origins and destinations that must be clustered. Several preliminary strategies were attempted in pursuit of this goal, including straightforward TAZ-based analysis and custom DBSCAN clustering. However, the results of these attempts were clumsy and did not provide a clear picture of where new transit routes would be most effective. To make the most of this novel granular data, a novel approach to optimizing trajectory clustering must be found, in order to identify corridors that have the highest potential for transit ridership. A successful deployment of that strategy would be invaluable to transit service and capital improvements planning.

\section{ACKNOWLEDGMENTS}

This report and the work described were sponsored by the U.S. Department of Energy (DOE) Vehicle Technologies Office (VTO) under the Big Data Solutions for Mobility Program, an initiative of the Energy Efficient Mobility Systems (EEMS) Program. The following DOE Office of Energy Efficiency and Renewable Energy (EERE) managers played important roles in establishing the project concept, advancing implementation, and providing ongoing guidance: David Anderson and Prasad Gupte. Data retrieved from Uber Movement, (c) 2019 Uber Technologies, Inc., https://movement.uber.com. Data @ OpenStreetMap contributors, http://www.openstreetmap.org/copyright

\section{REFERENCES}

[1] Gabriela Beirão and JA Sarsfield Cabral. 2007. Understanding attitudes towards public transport and private car: A qualitative study. Transport policy 14, 6 (2007), 
478-489.

[2] Cy Chan, Bin Wang, John Bachan, and Jane Macfarlane. 2018. Mobiliti: Scalable Transportation Simulation Using High-Performance Parallel Computing. In 2018 21st International Conference on Intelligent Transportation Systems (ITSC). 634-641. https://doi.org/10.1109/ITSC.2018.8569397

[3] Susan Handy, Lisa Weston, and Patricia L Mokhtarian. 2005. Driving by choice or necessity? Transportation Research Part A: Policy and Practice 39, 2-3 (2005)
183-203.

[4] Mizuki Kawabata and Qing Shen. 2007. Commuting inequality between cars and public transit: The case of the San Francisco Bay Area, 1990-2000. Urban Studies 44, 9 (2007), 1759-1780.

[5] Arnd König and Kay W Axhausen. 2002. The reliability of the transportation system and its influence on the choice behaviour. In Swiss transport research conference (strc), monte verità/ascona. 